\title{
Enhanced stability of vortex-antivortex states in two-component mesoscopic superconductors
}

\author{
R. Geurts, ${ }^{1}$ M. V. Milošević,,$^{1,2}$ J. Albino Aguiar, ${ }^{3}$ and F. M. Peeters ${ }^{1, *}$ \\ ${ }^{1}$ Departement Fysica, Universiteit Antwerpen, Groenenborgerlaan 171, B-2020 Antwerpen, Belgium \\ ${ }^{2}$ Departamento de Física, Universidade Federal do Ceará, 60455-900 Fortaleza, Ceará, Brazil \\ ${ }^{3}$ Departamento de Física, Universidade Federal de Pernambuco, Cidade Universitária, 50670-901 Recife-PE, Brazil
}

(Received 6 September 2012; revised manuscript received 7 November 2012; published 3 January 2013)

\begin{abstract}
Using the Ginzburg-Landau (GL) theory, we calculate the stability of sample symmetry-induced vortexantivortex molecules in a mesoscopic superconducting bilayer exposed to a homogeneous magnetic field. We demonstrate the conditions under which the two condensates cooperatively broaden the field-temperature stability range of the composite (joint) vortex-antivortex state. In cases when such broadening is not achieved, a reentrance of the vortex-antivortex state is found at lower temperatures. In a large portion of the phase diagram noncomposite states are possible, in which the antivortex is present in only one of the layers. In this case, we demonstrate that the vortex-antivortex molecule in one of the layers can be pinned and enlarged by interaction with a vortex molecule in the other. Using analogies in the respective GL formalisms, we map our findings for the bilayer onto mesoscopic two-band superconductors.
\end{abstract}

DOI: 10.1103/PhysRevB.87.024501

PACS number(s): 74.78.Na, 74.25.Dw, 74.25.Uv, 74.25.Wx

\section{INTRODUCTION}

Intuitively, one expects that the current of superconducting vortices in a homogeneous magnetic field can only turn in one particular direction, bundling the flux inside the vortex core. While true for bulk superconductors, this is not always the case in mesoscopic superconductors where confinement of the condensate plays an important role, and where the symmetry of the sample can stimulate the creation of one or more antivortices, i.e., vortices with currents circulating in the opposite direction, thus expelling flux. ${ }^{1}$ The existence of such vortex-antivortex ( $\mathrm{V}-\mathrm{Av})$ states can be understood from the Ginzburg-Landau (GL) theory close to the superconducting/normal state $(\mathrm{S} / \mathrm{N})$ boundary, where the GL equations for the superconducting order parameter become linear and the order parameter distribution must obey the symmetry of the sample. As a result, for, e.g., vorticity $L=3$ in a square sample, a state with a central antivortex surrounded by four vortices $(L=4-1=3)$ becomes the ground state. This particular V-Av state was studied extensively in Ref. 2, also further away from the $\mathrm{S} / \mathrm{N}$ boundary. The main findings were that the $\mathrm{V}$-Av state was highly sensitive to defects and imperfections, and that the $\mathrm{V}$-Av molecule is very compact (smaller than the coherence length $\xi$ ), both serious drawbacks regarding the experimental realization. To overcome these problems it was shown in Refs. 3 and 4 that nanoengineered pinning can make the $\mathrm{V}-\mathrm{Av}$ state more robust against imperfections and can multiply enlarge the $\mathrm{V}$-Av molecule. Nevertheless, direct observation of the V-Av state remains one of the main experimental challenges in mesoscopic superconductivity to date.

Notwithstanding the many attempts, the conceptually novel V-Av configuration has not been found experimentally. Therefore, there still exists a need for alternative proposals of a different system where this state can be stabilized in a substantially larger part of the field-temperature $(H-T)$ parameter space, with a clear separation of the vortices and the antivortex, and if possible, with some tunability of the V-Av state.
In this paper, we propose that the latter can be realized in mesoscopic superconducting bilayers, readily accessible in experiment. $^{5}$ Our system consists of two thin $(<\xi)$ squares of (in general) different superconducting materials, separated by an insulating layer that is sufficiently thin to allow for Josephson coupling between the two superconductors. This system can then be described by a Lawrence-Doniach Ginzburg-Landau (LDGL) model for just two superconducting layers. Due to the coupling between the superconducting layers and the different critical parameters and intrinsic length scales, nontrivial physical behavior is expected to emerge.

We here focus on the conditions in which the top layer induces the $\mathrm{V}-\mathrm{Av}$ in the bottom layer at higher temperatures, and vice versa - the bottom layer imprints the V-Av state on the top one at low temperatures. The main idea is that in such a case the temperature interval with stable $\mathrm{V}-\mathrm{Av}$ state can be substantially enlarged as compared to a regular mesoscopic sample. However, the Josephson and magnetic couplings between the layers add to the complexity of the competing interactions in this system. In the present paper, we describe the novel effects stemming from this competition.

The paper is organized as follows. In the next section, we outline the theoretical formalism used in the study, and give details of the considered sample geometry. In Sec. III, we demonstrate the obtained stability ranges of various found vortex-antivortex states, as a function of studied parameters. Sec. IV is subsequently devoted to the analysis of the influence of different parameters on the enhancement of the vortexantivortex state. In Sec. V, we translate our findings to a different system of recent interest-a two-band mesoscopic superconductor-after showing that the GL theoretical model for two-band superconductivity can be mapped onto the LDGL model for a bilayer system. We summarize our findings in Sec. VI.

\section{THEORETICAL FORMALISM AND SAMPLE PARAMETERS}

Our theoretical investigation is based on the LDGL theory ${ }^{6}$ in which the free energy of a very thin bilayer system (two 
superconducting layers of thickness $d$, separated by a spacer layer of thickness $s$ ) in a perpendicular magnetic field $H$ is expressed as ${ }^{7}$

$$
\begin{aligned}
F= & \int d \sum_{n=1}^{2}\left[\frac{1}{2 m_{n}}\left|\left(-i \hbar \vec{\nabla}-\frac{2 e}{c} \vec{A}\right) \Psi_{n}\right|^{2}\right. \\
& \left.+\alpha_{n}\left|\Psi_{n}\right|^{2}+\frac{1}{2} \beta_{n}\left|\Psi_{n}\right|^{4}\right] d S+s \int \Gamma\left|\Psi_{1}-\Psi_{2}\right|^{2} d S \\
& +\frac{1}{8 \pi} \int(\vec{\nabla} \times \vec{A}-\vec{H})^{2} d V,
\end{aligned}
$$

where $n=1,2$ indexes the layers. Here the last integral is taken over the entire space, whereas the first two are assumed uniform over the thickness superconducting and spacer layers, respectively (justified for thin layers), and integrated over surface projection of the layers. The constant $\Gamma$ quantifies the Josephson coupling between the superconducting layers, and is in general inversely proportional to the mass anisotropy of the tunneling Cooper pair and the squared interlayer spacing (assumed small here). Minimizing Eq. (1) with respect to $\Psi_{n}$ and $\vec{A}$ leads to the LDGL equations:

$$
\begin{aligned}
& \vec{\Pi}^{2} \psi_{1}-\left(\tau_{1}-\gamma-\left|\psi_{1}\right|^{2}\right) \psi_{1}-\gamma \delta \psi_{2}=0 \\
& \vec{\Pi}^{2} \psi_{2}-\alpha\left(\tau_{2}-\frac{\gamma}{m \alpha}-\left|\psi_{2}\right|^{2}\right) \psi_{2}-\frac{\gamma}{m \delta} \psi_{1}=0 \\
-\Delta \vec{A}= & \frac{1}{\kappa_{1}^{2}} \vec{j}_{1}[\Theta(-s / 2-z)-\Theta(-d-s / 2-z)] \\
& +\frac{\alpha}{\kappa_{2}^{2}} \vec{j}_{2}[\Theta(d+s / 2-z)-\Theta(s / 2-z)]
\end{aligned}
$$

where $\Theta(x)$ denotes the Heaviside step function, and $z=0$ is taken in the center of the sample. Equations (2) are written in dimensionless form, with $\psi_{n}=$ $\Psi_{n} / \Psi_{n 0}, \Psi_{n 0}=\sqrt{-\alpha_{n 0} / \beta_{n}}, \alpha=m_{2} \alpha_{20} / m_{1} \alpha_{10}, m=m_{1} / m_{2}$, $\delta=\sqrt{\alpha_{20} \beta_{1} / \alpha_{10} \beta_{2}}=\Psi_{20} / \Psi_{10}, \quad \gamma=\Gamma s / \alpha_{10} d$, and $\tau_{n}=$ $-\ln \left(T / T_{c n}\right)\left(T_{c n}\right.$ are the critical temperatures of the layers, when decoupled). ${ }^{8} \vec{\Pi}=-i \vec{\nabla}-\vec{A}$ is the gauge-invariant momentum, with vector potential scaled by $\hbar c / 2 e \xi_{10}=\phi_{0} / 2 \pi \xi_{10}$ ( $\phi_{0}$ being the flux quantum). Here $\xi_{10}=\hbar / \sqrt{-2 m_{1} \alpha_{10}}$ denotes the coherence length of the first layer at zero temperature, and also serves as unit of all distances. $\kappa_{n}$ are the GL parameters of the uncoupled layers, defined for each layer independently as the ratio of respective $\lambda$ (penetration depth) and $\xi$ at zero temperature.

The equations for $\psi_{n}$ are solved in $2 \mathrm{D}$, and the supercurrents $j_{n}$ in Eq. (2) are also calculated in 2D as $\vec{j}_{n}=$ $\mathcal{R}\left[\psi_{n}^{*}(-i \stackrel{\vec{\nabla}}{\nabla} \vec{A}) \psi_{n}\right]$ (where $\mathcal{R}$ stands for the real part). However, the equation for $A$ in Eq. (2) is solved in $3 \mathrm{D}$. We then solve the system of Eqs. (2) self-consistently, with the Neumann boundary condition (imposing no supercurrent can pass through the sample boundary) and by mapping it on a regular square grid. Details on the numerical procedure can be found in Ref. 9.

Without loss of generality, we consider a sample of square shape. In order to have an optimally enlarged V-Av molecule, ${ }^{4}$ we will use our previously proposed geometry with $2 \times 2$ holes (unless stated otherwise). All the obtained results can anyhow be extrapolated to the case of a plain square, as the symmetry of the sample, and thus the physical origin of the V-Av state therein, is the same. The size (side) of the sample will be further denoted by $w$. The holes are of square shape with side $w_{h}$, and are centered in the sample at a distance $2 d_{h}$ from each other [see Fig. 2(b)]. In what follows, we consider a sample with $w=10 \xi_{10}, w_{h}=1.5 \xi_{10}$, and $d_{h}=1.75 \xi_{10}$, with each layer having thickness $1.0 \xi_{10}$. After scaling all temperatures to $T_{c 1}$, we are left with six parameters to fully characterize the superconducting properties: the ratio of critical temperatures of the layers $T_{c 2} / T_{c 1}$, the ratio of the coherence lengths of two layers at zero temperature $\alpha$, the ratio of the Cooperpair densities in the absence of any external influence $\delta$, strength of Josephson coupling $\gamma$, mass ratio $m$, and the GL parameter $\kappa_{1}\left(\kappa_{2}\right.$ is then fixed by the choice of the preceding parameters).

\section{STABILITY RANGE OF THE V-Av STATES}

Without mutual coupling, the two superconductors in the bilayer are independent of each other, and in zero field superconductivity in them would cease at respective temperatures $T_{c n}$. In this case, one can apply the earlier findings, and conclude that the $L=4-1 \mathrm{~V}-\mathrm{Av}$ state should be found directly below the $\mathrm{S} / \mathrm{N}$ boundary $\left[T_{c}(H)\right]$ and should extend down to temperatures at which the side of the sample measures $\sim 7 \xi(T) .{ }^{4}$ However, the two layers can be of different materials having very different length scales and critical temperatures, so their V-Av stability regions-in the absence of coupling-would be displaced in the magnetic flux-temperature $(\phi-T)$ diagram, as shown in the left column of Fig. 1. In Figs. 1(a)-1(c), we increase $T_{c 2}$, so that the V-Av stability region of the second layer shifts up in temperature following the shift of the S/N boundary of that layer.

In the presence of Josephson coupling, one immediately assumes that the superconductor dominant in superconducting
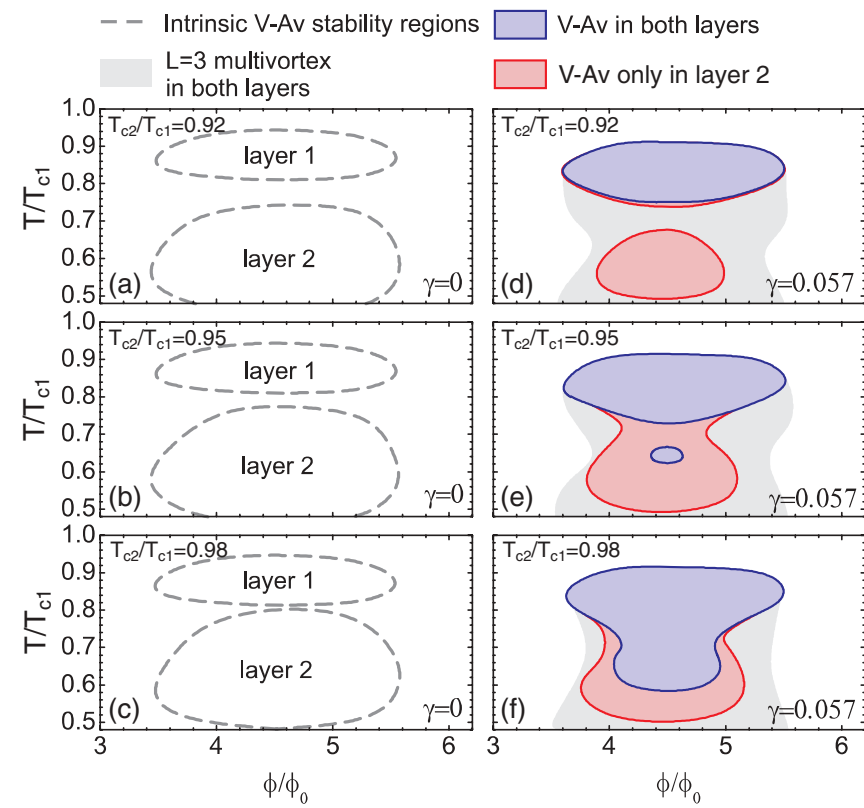

FIG. 1. (Color online) $\phi-T$ stability regions of the $L=4-1$ $\mathrm{V}$-Av state in a bilayer square with $2 \times 2$ holes, in the absence of Josephson coupling (left) and for nonzero coupling (right). The parameters other than shown are $\alpha=0.3, \delta=9$, and $\kappa_{n} \gg 1$. 
properties should impose its vortex configuration on the weaker superconductor, and this is certainly the case for $T>T_{c 2}(H)$, where the first layer is in the V-Av state, and the second layer superconducts solely due to the coupling. However, at lower temperatures the second layer hosts the $\mathrm{V}-\mathrm{Av}$ state, and in order to impose it on the first layer one needs $\delta \gg 1$, as that parameter regulates the influence of $\psi_{2}$ on $\psi_{1}$ in Eq. (2). Therefore, if the latter condition is met, the superconducting condensate in a square bilayer can potentially sustain the V-Av state to far lower temperatures compared to the case of separate layers. To ideally extend the stability region of the joint $\mathrm{V}-\mathrm{Av}$ state, the lower boundary of the $\mathrm{V}-\mathrm{Av}$ stability region in the first layer should be close to the upper boundary of the $\mathrm{V}-\mathrm{Av}$ region in the second layer, i.e., around $T_{c 2}(H)$. When the $\mathrm{V}$-Av state in the second superconductor is found at much lower temperatures than the one of the first superconductor [see Fig. 1(d)], or for insufficiently strong Josephson coupling, no enlargement of the stability region of the V-Av state is realized. Instead, one finds only at high temperatures an island of the composite $V$-Av state (i.e., a state where the $\mathrm{V}-\mathrm{Av}$ molecule exists in both superconductors). As temperature is lowered, the $L=4-1$ state disappears and reappears only in the second layer, while the vortex state in the first superconductor is a simple $L=3$ noncircular symmetric multivortex [shown in Fig. 2(b)]. When not every (anti)vortex in one layer is matched by a (anti)vortex in the other layer, we refer to such a state as a noncomposite one.

In Fig. 1(e), with $T_{c 2}$ increased as compared to Fig. 1(d), one observes two separate regions of the composite V-Av state-the top region induced by the first layer and the bottom region due to the second layer. As a unique property of our bilayer system, we predict a reentrance of the composite V-Av state by sweeping temperature up or down. When further increasing $T_{c 2}$, Fig. 1(f) shows that the stability regions of the $\mathrm{V}$-Av states in the two superconductors merge into a large region of a composite $\mathrm{V}$-Av state. A similar effect is found with increasing Josephson coupling [see Fig. 2(a)], where the composite $\mathrm{V}$-Av state expands at the expense of the noncomposite antivortex state $(L=3$ in first layer and $L=4-1$ in the other). However, the latter state is also an interesting one, since it involves an antivortex in just one of the layers. In Fig. 2(b) we show the Cooper-pair density plots of $\psi_{1}$ and $\psi_{2}$ respectively for such a state, and location of vortices and an antivortex can be better visualized in the circulation of phase of the order parameters shown in Fig. 2(c). Remarkably, this state is asymmetric; i.e., the antivortex in the second layer is not centered. This is a consequence of the interaction of the $\mathrm{V}$-Av molecule with an asymmetric $L=3$ multivortex state (one hole without vorticity) in the first layer. Notably, as already shown in Figs. 1 and 2, this asymmetric state can be stable in a rather large portion of the $\phi-T$ parameter space. One should note that this state as a whole is fourfold degenerate in energy (can be rotated by integer multiples of $90^{\circ}$ due to the sample symmetry), but is reproducible in every other respect.

Finally, in the analysis of Fig. 2(a), we briefly point out the observed decrease of the top critical temperature of the composite V-Av state. This is due to the increased Josephson coupling, which enters the temperature-dependent terms in Eq. (2) and gradually decreases the overall $T_{c}$ of the sample to the average between the two critical temperatures of the two layers.
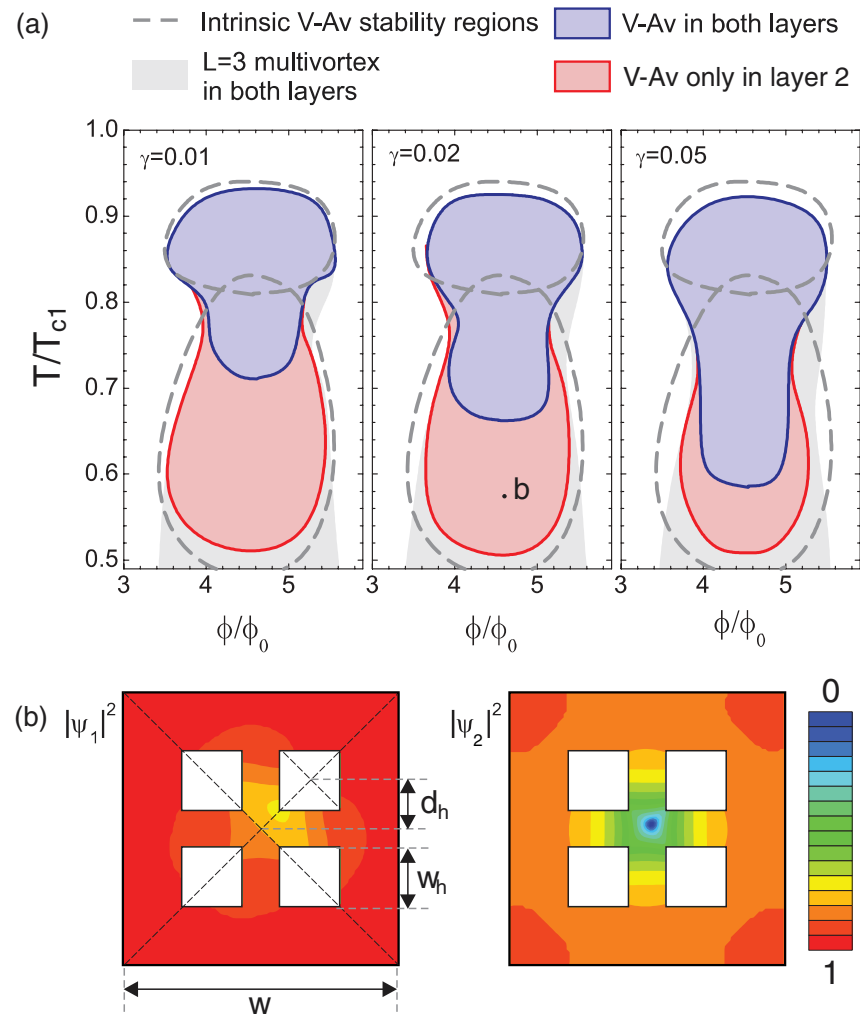

(c)
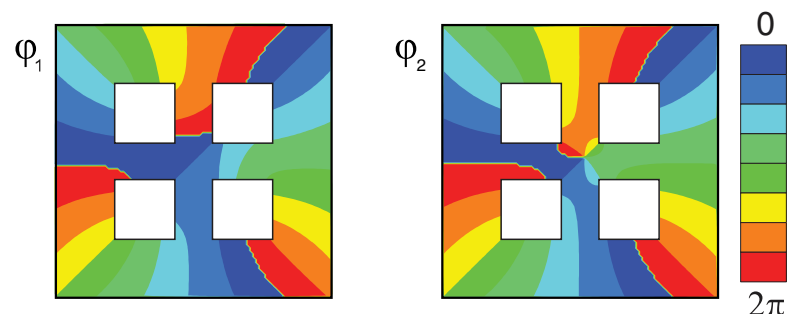

FIG. 2. (Color online) (a) $\phi$ - $T$ stability regions of the $L=4-1$ $\mathrm{V}$-Av state in a $2 \times 2$ perforated square bilayer, for increasing Josephson coupling. Other parameters are $\alpha=0.3, \delta=9$, and $T_{c 2}=T_{c 1}$. (b) The Cooper-pair density in the two layers for the noncomposite vortex-antivortex state, marked by a point in diagram (a). (c) The distribution of phase of the order parameters shown in (b), facilitating visualization of (anti)vortices.

\section{OTHER CONDITIONS FOR ENHANCED V-Av STATE}

In mesoscopic superconductivity, one of the key parameters is the coherence length of the Cooper-pair condensate. Therefore, in Fig. 3, we show the calculated V-Av $\phi$ - $T$ stability regions for increasing parameter $\alpha$, i.e., decreasing the nominal coherence length of the second layer $\left(\xi_{2}\right)$. We observe that by increasing $\alpha$, the stability region of the composite $\mathrm{V}$-Av state first expands, but subsequently shrinks as $\alpha$ approaches unity. Namely, as $\xi_{2}$ decreases, the intrinsic critical temperature of the second layer at zero field $T_{c 2}$ does not change, but its value for a given field increases [i.e., $T_{c 2}(H)$ boundary tilts upwards]. This moves the intrinsic $\mathrm{V}-\mathrm{Av}$ stability regions in the two layers closer together which enables their merging into a larger stability region for the composite V-Av state, similarly to what is shown in Fig. 1. However, at the same time, decreasing $\xi_{2}$ increases the effective size of the sample, 

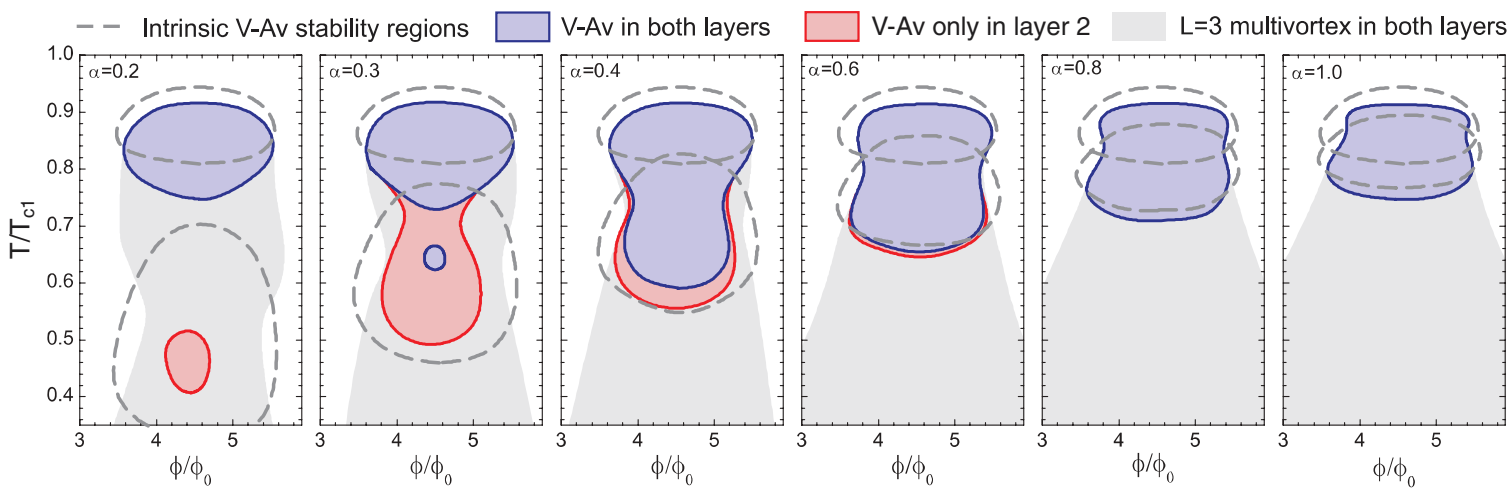

FIG. 3. (Color online) The evolution of the stability region of the composite $L=4-1 \mathrm{~V}$-Av state in a $2 \times 2$ perforated square bilayer with the increasing parameter $\alpha$ (decreasing $\xi_{2} / \xi_{1}$ ). Other parameters are $\gamma=0.057, \delta=9$, and $T_{c 2}=0.95 T_{c 1}$.

and a higher temperature is needed to enhance the effect of confinement and induce the $\mathrm{V}$-Av state in the second layer. This eventually shrinks the $\phi-T$ stability region of the V-Av state in the second layer, consequently of the composite state as well.

When the layer with the lower critical temperature is able to electronically influence the other layer more strongly than the other way around, it will result in a substantial extension of the composite $\mathrm{V}-\mathrm{Av}$ state in a bilayer system. The layer with lower $T_{c}$ therefore needs to have a larger Cooper-pair density. For $T_{c 1}>T_{c 2}$, we thus need $\delta \gg 1$. The influence of $\delta$ is clearly illustrated in Fig. 4. In panels (a) and (b) $\delta$ is small, so the influence of the first layer on the second is strong. As a consequence, the vortex state of the first layer is imprinted on the second one, and the intrinsic V-Av state of the second layer is almost completely destroyed. In Fig. 4(c), where $\delta$ is larger, the $\mathrm{V}$-Av in the second layer revives. It is however not strong enough to induce the $\mathrm{V}$-Av state in the first layer in its full $\phi-T$ stability region. When $\delta$ is increased further, as in Fig. 4(d), the second layer is finally able to impose the V-Av state on the first layer in a larger portion of the $\phi-T$ space.

As a last part of the discussion, we point out the importance of the magnetic coupling between vortices in the two layers. Even in a sample without holes, a noncomposite antivortex

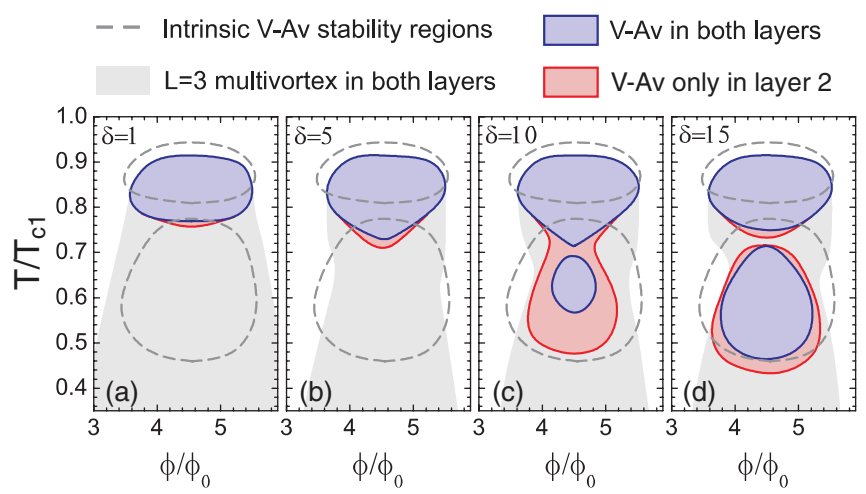

FIG. 4. (Color online) The evolution of the stability region of the composite $L=4-1 \mathrm{~V}$-Av state in a $2 \times 2$ perforated square bilayer with the increasing $\delta$ (the ratio of Cooper-pair density in the two layers at zero temperature and zero magnetic field). Other parameters are $\gamma=0.057, \alpha=0.3$, and $T_{c 2}=0.95 T_{c 1}$. state can be realized-such as the one shown in Fig. 5. In this case, we have the $L=4$ state in one layer, with four separated vortices in a multivortex state, and the $L=4-$ $1 \mathrm{~V}$-Av state in the second layer. While the vortices in the first layer adopt a fourfold-symmetric position due to their mutual repulsion, the vortex state in the second layer becomes fourfold symmetric due to the magnetic field generated by the first layer. Moreover, the vortices of the first layer now act as magnetic pinning centers for the vortices in the second layer, similarly to the influence of $2 \times 2$ holes, and help to realize a much expanded $\mathrm{V}$-Av molecule in a plain square with $\mathrm{V}$-Av distance of $\sim 1.4 \xi_{1}$ (in Ref. 1, this distance was below the coherence length). Notice that this effect does not strictly depend on sample geometry, as four vortices in the $L=4$ state form a square configuration even in a sufficiently large circular superconducting disk. ${ }^{10}$

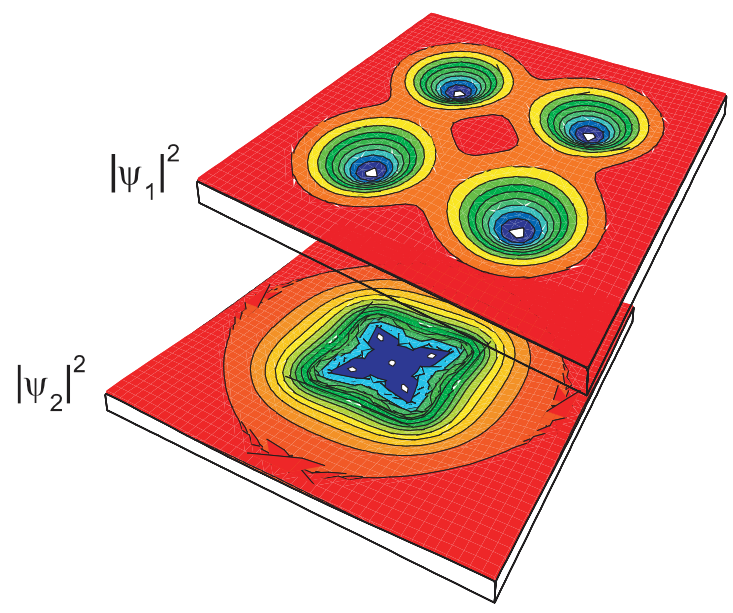

FIG. 5. (Color online) Contour plots of the Cooper-pair density of the first (top) and the second layer (bottom) for the $L=4 / L=4-1$ state in a mesoscopic bilayer square (no holes), demonstrating the $\mathrm{V}$ Av molecule in the second layer which is pinned by the vortices of the first layer through only magnetic coupling. The parameters used are $w=16 \xi_{10}, \alpha=0.25, \gamma=0, \delta=0.45, \phi / \phi_{0}=5, T=0.6 T_{c 1}, \kappa_{1}=$ 0.5 , and $\kappa_{2}=0.555$. The interlayer distance is greatly exaggerated in the figure, to enable visualization of the vortex states in both layers. 


\section{V-Av MOLECULE IN TWO-BAND MESOSCOPIC SUPERCONDUCTORS}

The past decade has seen a large scientific attention on superconducting $\mathrm{MgB}_{2}$. Among other things, this material possesses two superconducting gaps. ${ }^{11-14}$ In other words, it hosts two superconducting condensates, and their mutual coupling (e.g., Josephson and/or magnetic one), in combination with different intrinsic length scales and temperaturedependent properties, makes the net behavior highly nontrivial. Furthermore, the effect of the boundary in the mesoscopic regime can be very different for the two condensates, and can lead to novel physics. For example, noncomposite vortices (with cores displaced in the two bands) and fractional vortices (with noninteger net flux) have been predicted in mesoscopic two-band disks. ${ }^{15-17}$

We will show that this system has similarities to the superconducting bilayer studied in the preceding sections of this paper. To make this analogy clearer, we write here the so-called two-component (TC) GL theory, which reduces to the dimensionless equations 15,18

$$
\begin{gathered}
\vec{\Pi}^{2} \psi_{1}-\left(\chi_{1}-\left|\psi_{1}\right|^{2}\right) \psi_{1}-\gamma \psi_{2}=0, \\
\vec{\Pi}^{2} \psi_{2}-\alpha\left(\chi_{2}-\left|\psi_{2}\right|^{2}\right) \psi_{2}-\alpha \frac{n_{1}}{n_{2}} \gamma \psi_{1}=0, \\
-\Delta \vec{A}=\frac{1}{\kappa_{1}^{2}} \vec{j}_{1}+\frac{\alpha}{\kappa_{2}^{2}} \vec{j}_{2} .
\end{gathered}
$$

In these equations the order parameters are expressed in $W$ [defined as $W^{2}=8 \pi^{2} T_{c}^{2} / 7 \zeta(3)$ ], lengths are measured in $\xi_{1}=\hbar^{2} v_{1}^{2} / 6 W^{2}$ (which now has the meaning of the coherence length of the condensate in the first band, at zero temperature, as if it was decoupled from the system), and the vector potential in $A_{0}=2 \pi \xi_{1} / \phi_{0} . v_{i=1,2}$ are the Fermi velocities in the two bands which define the parameter $\alpha=\left(v_{1} / v_{2}\right)^{2}$, a measure for the squared ratio of the intrinsic coherence lengths in two bands (analogously to the bilayer case). The temperature dependence is captured by $\chi_{i}=S_{i} /\left(n_{i} \eta\right)-\ln \left(T / T_{c}\right)$, with $S_{1}=\lambda_{22}-n_{1} \eta S, \quad S_{2}=\lambda_{11}-n_{2} \eta S, \quad S=\left(n_{1} \lambda_{11}+n_{2} \lambda_{22} \pm\right.$ $\left.\sqrt{\left(n_{1} \lambda_{11}-n_{2} \lambda_{22}\right)^{2}+4 n_{1} n_{2} \lambda_{12}^{2}}\right) /\left(2 n_{1} n_{2} \eta\right)$, and $\eta=\operatorname{det} \Lambda=$ $\lambda_{11} \lambda_{22}-\lambda_{12}^{2} . \Lambda=\left|\begin{array}{cc}\lambda_{11} & \lambda_{12} \\ \lambda_{21}=\lambda_{12} & \lambda_{22}\end{array}\right|$ is the (symmetric) coupling matrix, using the notation of Ref. 19. From the above expressions one can obtain the intrinsic critical temperatures, i.e., the temperatures at which the gaps would deplete in the absence of coupling: $T_{c i}=T_{c} \exp \left(-S_{i} / n_{i} \eta\right)$, again adding to similarities to the bilayer case, where each layer has its own $T_{c}$ in the absence of coupling. $\gamma=\lambda_{12} / n_{1} \eta$ determines the strength of the Josephson-like coupling between the band condensates. $n_{i}$ represent the partial density of states in the two bands (preserving $n_{1}+n_{2}=1$ ). The intrinsic GL parameters of each band can be defined as $\kappa_{1}^{2}=\pi / 2 n_{1} N(0) W^{2} \phi_{0}^{2}$ and $\kappa_{2}^{2}=\kappa_{1}^{2} \alpha^{2} n_{1} / n_{2}$. Summarizing, we have eight parameters to fully characterize the superconducting properties: $v_{1}$ (which only enters the scaling of sizes/distances), $\alpha, n_{1}, \lambda_{11}, \lambda_{12}, \lambda_{22}$, $W$ (setting $T_{c}$ ), and $N(0)$ (entering $\kappa_{i}$ ). The supercurrents $j_{i}$ in Eq. (3) are defined as $\vec{j}_{i}=\mathcal{R}\left[\psi_{i}^{*}(-i \nabla-\vec{A}) \psi_{i}\right]$, again the same as in Eq. (2) for the bilayer system.

With the Neumann boundary condition that no supercurrent can pass through the boundary of the sample, we solve this system of equations self-consistently on a square Cartesian grid, for the exact same size and geometry of the sample used for the bilayer in Sec. II. The boundary conditions on the supercurrent in this calculation have an additional important consequence, as they strongly couple the TCGL equations and enhance the difference in spatial profiles of two order parameters. For that reason, we preserve the higher order terms in the GL model, in spite of the issues pointed out in Ref. 19. Although incomplete, those terms are essential for the description of the involved physics in the mesoscopic two-band superconductors, and are also instrumental to the analogy to bilayers-which is our main goal in this section. Namely, one can immediately notice the striking resemblance of Eqs. (2) and (3). The equations for the order parameters have the same shape, though somewhat different coefficients, while the equations for the magnetic field are identical in the two cases (of course, after rescaling to different respective units). Motivated by this, in what follows, we examine whether similar physics to that reported for bilayers in Secs. III and IV holds for two-band samples of the same size and geometry. This is not directly clear, since for example Josephson coupling multiplies both order parameters in LDGL equations, and that with opposite signs. In TCGL, parameters $\chi$ and $\gamma$ are interdependent via microscopic parameters, and in a rather complicated manner. Another important difference can be that the coupling term in the second equation of LDGL does not directly depend on the ratio of the length scales of the two components, whereas in TCGL it does.

\section{A. Influence of the microscopic parameters of the bands}

Without mutual coupling, the two bands are independent and superconductivity in them ceases at their respective temperatures $T_{c i}$. Therefore, the complete discussion from the beginning of Sec. III applies to two-band samples as well. We therefore construct the applied magnetic flux-temperature $(\phi-T)$ phase diagram in Fig. 6, in analogy to Fig. 1. From top to bottom $\lambda_{22}$ is increased, and with it the critical temperature of the second band $T_{c 2}$, with the effect that the V-Av stability region of the second band is shifting up in temperature. This picture is of course unrealistic - any introduced coupling between bands will also couple the $\mathrm{V}$-Av stability regions (see the right panel of Fig. 6). In comparison with Fig. 1, we notice the very similar behavior of the stability range of $\mathrm{V}-\mathrm{Av}$ molecules, as well as the noncomposite states. For $T>T_{c 2}$, the first band mostly imposes its V-Av state on the second band. To extend the stability region of the V-Av state to lower temperatures $\left(T<T_{c 2}\right)$, the reverse should be enabled; i.e., one needs $\alpha n_{1} / n_{2} \ll 1$, as this multiplying factor in Eq. (3) ensures that the influence of $\psi_{2}$ on $\psi_{1}$ is much stronger than the opposite. In Fig. 6, we used $\alpha n_{1} / n_{2}=0.0125$. The interband coupling was introduced via the nonzero $\lambda_{12}$. Considering other constants in the $\Lambda$ matrix, the choice of $\lambda_{11}=23$ in Fig. 6 may seem unrealistic. However, in the other notation from the literature $\lambda_{\mu \nu}^{\text {(lit) }}=n_{\mu} \lambda_{\mu \nu}$, one has $\lambda_{11}^{\text {(lit) }}=0.92, \lambda_{12}^{\text {(lit) }}=0.015$, $\lambda_{21}^{\text {(lit) }}=0.36$, and $\lambda_{22}^{\text {(lit) }}=0.77$ for the middle panel of Fig. 6 . These values are of the same order as the ones readily found in the literature. ${ }^{20-23}$ In this notation, we can express in a different way the criterion to obtain an extended V-Av region in the $\phi-T$ space. Namely, $\lambda_{12}^{\text {(lit) }}<\lambda_{21}^{\text {(lit) }}$ directly means that the 


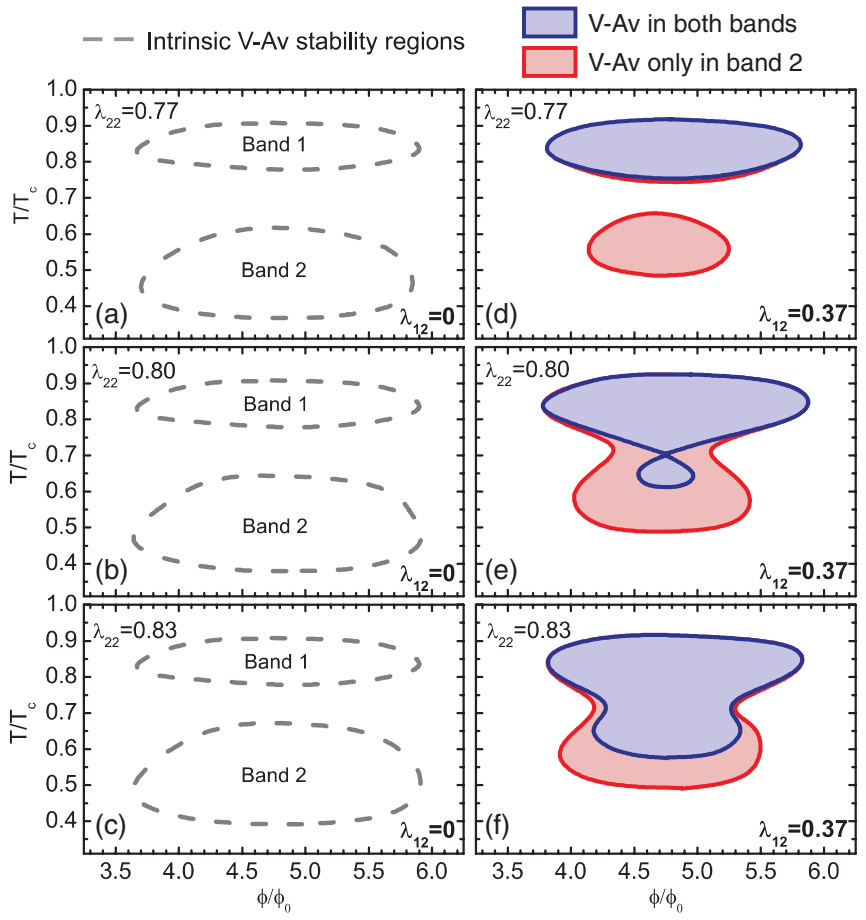

FIG. 6. (Color online) $\phi-T$ stability regions of the $L=4-1$ $\mathrm{V}$-Av state in a two-band square with $2 \times 2$ holes, in the absence of interband coupling (left) and for nonzero coupling (right). The parameters other than shown in the figures are $\alpha=0.3, \lambda_{11}=23$, $n_{1}=0.04$, and $\kappa_{i} \gg 1$.

second band has a stronger influence on the first band than the other way around.

Let us now discuss the influence of the coupling between the bands based on the results shown in Fig. 7. As the interband coupling is increased (by increasing $\lambda_{12}$ ), the influence of the second condensate on the first one gradually increases as the

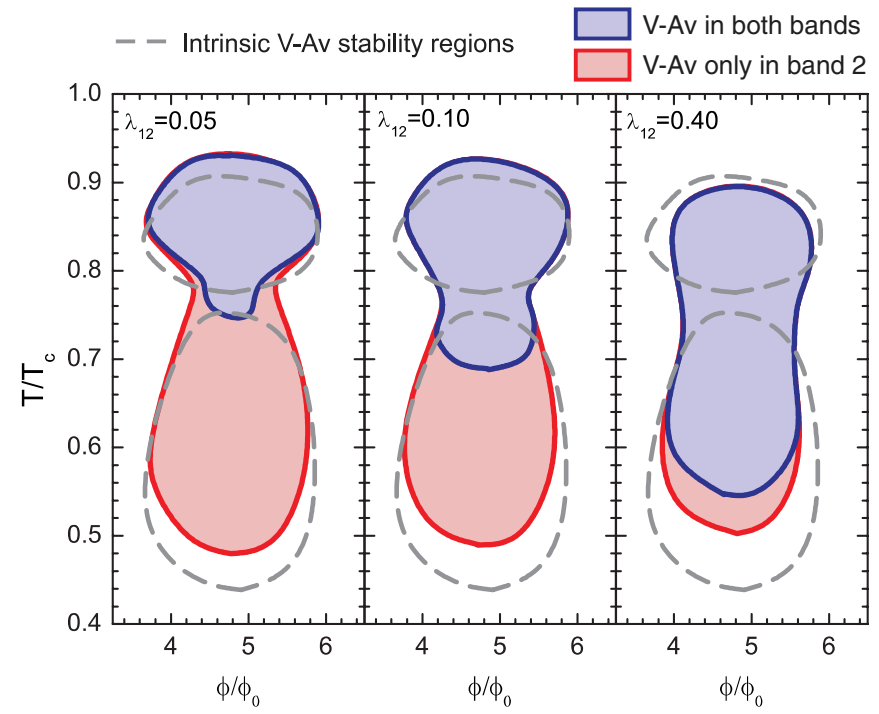

FIG. 7. (Color online) The stability regions of the $L=4-1$ $\mathrm{V}-\mathrm{Av}$ state in a $2 \times 2$ perforated two-band square, as a function of increasing interband coupling (via $\lambda_{12}$ ). Other parameters are $\alpha=0.3, \lambda_{11}=23, \lambda_{22}=0.94, n_{1}=0.04$, and $\kappa_{1} \gg 1$.

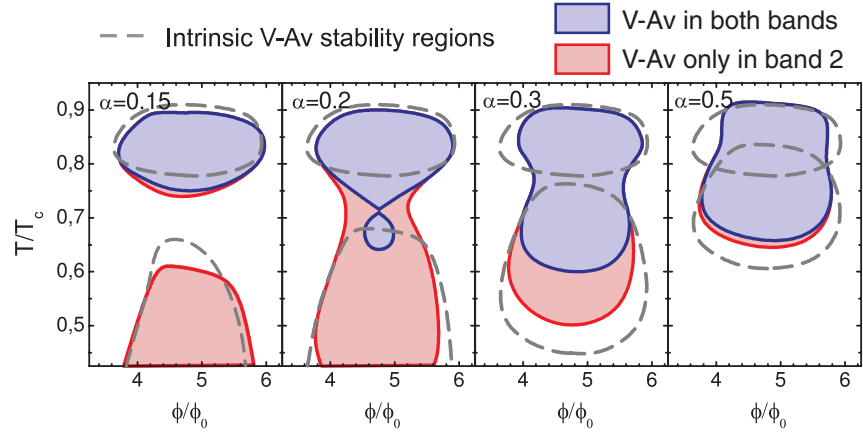

FIG. 8. (Color online) The evolution of the stability region of the composite $L=4-1 \mathrm{~V}$-Av state in a $2 \times 2$ perforated two-band square with the increasing $\alpha$ and thus decreasing Fermi velocity and coherence length of the second band. Other parameters are $n_{1}=0.04$, $\lambda_{11}=23, \lambda_{12}=0.2$, and $\lambda_{22}=0.96$.

composite state annexes a larger part of the V-Av state stability region of the second band. At the same time, the stability region of the noncomposite $\mathrm{V}$-Av state is reduced, but at a slower rate since the influence of the first band on the second one is weaker than the opposite. With further increasing coupling, we observe that the composite V-Av state expands further at the expense of the noncomposite state (which finally disappears). During this process, we notice a decrease of the critical temperature for the V-Av state in Fig. 7, similarly to that observed for bilayers in Fig. 2. However, the origin is slightly different-in the present case increasing $\lambda_{12}$ increases the coupling constant $\gamma$-but also induces a strong decrease of the intrinsic critical temperatures $T_{c i}$ which all combine to a decreasing overall $T_{c}$.

Following further the reasoning presented in Sec. IV, we now discuss the influence of the length scales of the two-band condensates. The difference between those lengthscales was recently thoroughly discussed in literature, ${ }^{24}$ and is known to potentially lead to new physical phenomena, ${ }^{25}$ while being particularly pronounced close to the hidden critical point of two-band superconductors. ${ }^{26}$ In Fig. 8, we show the calculated $\phi-T$ phase diagrams for increasing parameter $\alpha$, in a $2 \times 2$ perforated two-band square. Changing this parameter in TCGL corresponds to changing the Fermi velocity $v_{2}$ and consequently changing the intrinsic coherence length $\xi_{2}$ of the second band, while $\xi_{1}$ remains constant. It then comes as a little surprise that the results of Fig. 8 are very similar to our results for a bilayer in Fig. 3, since the LDGL parameter $\alpha$ has the same meaning. Actually, all features observed in Fig. 3 are reproduced in Fig. 8. However, one subtle difference exists, since in the TCGL model increasing $\alpha$ also strengthens the influence of the first band on the second one [see Eq. (3)]. This contributes to the faster reduction of the stability region of the $\mathrm{V}$-Av state with increasing $\alpha$ from intermediate values to unity.

After the phenomenon described in our analysis of bilayers in Sec. III, we emphasize here once more the possible reentrant behavior of the $\mathrm{V}$-Av state as a function of temperature, observed also in the two-band sample. For example, in Fig. 8, for $\alpha=0.2$ we notice two separate islands of the composite $\mathrm{V}$-Av state, observable by, e.g., changing symmetry of the magnetic field profile obtained by scanning Hall probe imaging at different temperatures. ${ }^{27}$ For $\alpha=0.15$ two separate islands 


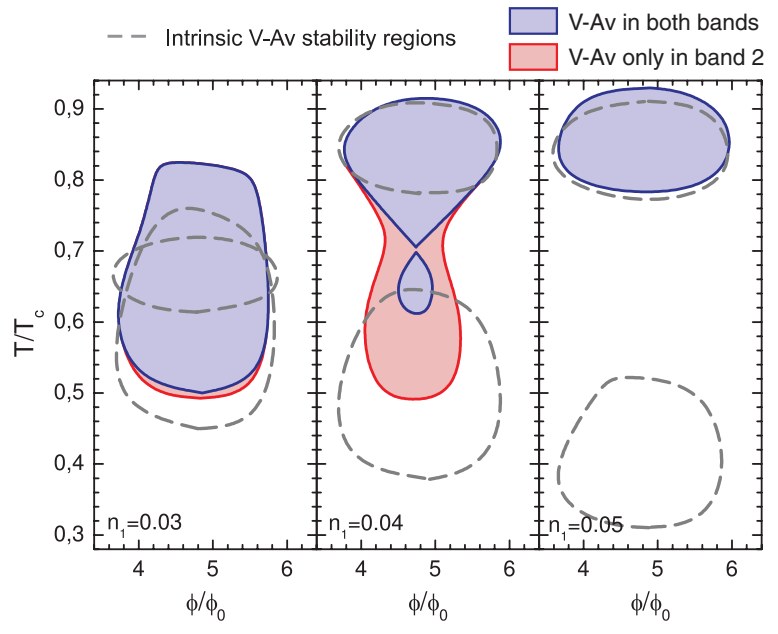

FIG. 9. (Color online) Stability regions in $\phi-T$ parameter space of the $L=4-1 \mathrm{~V}$-Av state in a $2 \times 2$ perforated two-band square for different local density of states $n_{1}$ (and correspondingly changing $n_{2}$ ). Other parameters are $\alpha=0.3, \lambda_{11}=23, \lambda_{12}=0.37$, and $\lambda_{22}=0.83$.

of the V-Av state are found only in the second band, which may be verified experimentally by imaging only the second band (as is readily done for the $\pi$ band of $\mathrm{MgB}_{2}$; see Ref. 28). The splitting/merger of the stability regions of the V-Av state in two bands can also be realized by control of the local density of states, which might be achieved to certain extent by local carrier injection. ${ }^{29}$ As explained earlier, for a broad $\phi-T$ stability of the composite V-Av state one needs a low $\alpha n_{1} / n_{2}$ factor, which can be realized by a small $n_{1}$. Increasing $n_{1}$ increases $T_{c 1}$ and decreases $T_{c 2}$, and thus results in a gradual separation of this region into two, as we show in Fig. 9. Increasing $n_{1}$ also diminishes the effect of the second band on the first one, and the composite $\mathrm{V}$-Av state can no longer be found at low temperatures (although present in the second band). As a result, for, e.g., $n_{1}=0.04$ in Fig. 9, we observe the reentrance of the composite $\mathrm{V}$-Av state with changing temperature, whereas for larger $n_{1}$ the reentrance was found for the V-Av state only in the second band.

\section{CONCLUSIONS}

In summary, we demonstrated that the V-Av state can be stabilized in mesoscopic single-band superconducting bilayers and two-band samples in a far larger portion of the field-temperature phase diagram than is the case for single mesoscopic polygons. We also showed that $\mathrm{V}-\mathrm{Av}$ states in two-component samples are much richer than their single-component counterparts, and appear in composite and noncomposite (asymmetric) form, either of which can show reentrant behavior as a function of temperature. This is observable through, e.g., the symmetry of the magnetic field profile measurable by scanning Hall probe microscopy. ${ }^{27}$ Alternatively, the vortex state in either component can also be directly imaged by, e.g., scanning tunneling microscopy. Although this work was motivated by possibly improved conditions for the observation of the $\mathrm{V}-\mathrm{Av}$ molecule in mesoscopic samples, and strengthening of the molecule itself, it is clear from our results that more studies of bilayer systems are needed-related to rich possible vortex phases, but also to their dynamics in applied drive to one or both layers, as well as the flux cutting phenomena of relevance to layered bulk superconductors such as high- $T_{c}$ ones. Likewise, more work should be done on two-band samples, since our results reiterate the possibility of vortex decomposition in such samples (reported earlier in the literature; see Refs. 15,16 and citing articles), which is bound to generate exciting new physics in both static and dynamic regimes. Unfortunately, in spite of the recent resurgence of interest in multiband materials (mainly due to iron pnictides), almost no work has been experimentally done to date on mesoscopic samples.

\section{ACKNOWLEDGMENTS}

This work was supported by the Flemish Science Foundation (FWO-Vlaanderen), the Brazilian science agencies FACEPE/CNPq under Grant No. APQ-0589-1.05/08 and CNPq under Grant No. 309832/2007-1, and the CNPq-FWO cooperation program under Grant No. 490681/2010-7. M.V.M. acknowledges support from the CAPES-PVE program. *francois.peeters@ua.ac.be

${ }^{1}$ L. F. Chibotaru, A. Ceulemans, V. Bruyndoncx, and V. Moshchalkov, Nature (London) 408, 833 (2000).

${ }^{2}$ J. Bonča and V. V. Kabanov, Phys. Rev. B 65, 012509 (2001); A. S. Mel'nikov, I. M. Nefedov, D. A. Ryzhov, I. A. Shereshevskii, V. M. Vinokur, and P. P. Vysheslavtsev, ibid. 65, 140503 (2002); T. Mertelj and V. V. Kabanov, ibid. 67, 134527 (2003).

${ }^{3}$ C. Carballeira, V. V. Moshchalkov, L. F. Chibotaru, and A. Ceulemans, Phys. Rev. Lett. 95, 237003 (2005).

${ }^{4}$ R. Geurts, M. V. Milošević, and F. M. Peeters, Phys. Rev. Lett. 97, 137002 (2006); Phys. Rev. B 75, 184511 (2007).

${ }^{5}$ H. Bluhm, N. C. Koshnick, M. E. Huber, and K. A. Moler, Phys. Rev. Lett. 97, 237002 (2006).

${ }^{6} \mathrm{~W}$. Lawrence and S. Doniach, in Proceedings of the Twelfth International Conference on Low Temperature Physics, edited by E. Kanda (Academic Press of Japan, Kyoto, 1971), pp. 361-362.
${ }^{7}$ Notice that the original Lawrence-Doniach theory is proposed for the case of parallel magnetic field; hence the coupling between layers in our case has no phase factor (perpendicular component of the vector potential is zero).

${ }^{8}$ Logarithmic temperature dependence is taken ad hoc in an attempt to improve the validity of the results at lower temperatures. However, taking standard first-order linear temperature dependence in the GL theory would not change the conclusions of the paper.

${ }^{9}$ M. V. Milošević and R. Geurts, Physica C 470, 791 (2010); for iterative procedure, see V. A. Schweigert and F. M. Peeters, Phys. Rev. B 57, 13817 (1998).

${ }^{10}$ B. J. Baelus and F. M. Peeters, Phys. Rev. B 65, 104515 (2002).

${ }^{11}$ W. Pickett, Nature (London) 418, 733 (2002).

${ }^{12}$ J. Nagamatsu, N. Nakagawa, T. Muranaka, Y. Zenitani, and J. Akimitsu, Nature (London) 410, 63 (2001). 
${ }^{13}$ C. Buzea and T. Yamashita, Supercond. Sci. Technol. 14, R115 (2001).

${ }^{14}$ X. X. Xi, Rep. Prog. Phys. 71, 116501 (2008).

${ }^{15}$ R. Geurts, M. V. Milošević, and F. M. Peeters, Phys. Rev. B 81, 214514 (2010).

${ }^{16}$ L. F. Chibotaru, V. H. Dao, and A. Ceulemans, Europhys. Lett. 78, 47001 (2007); L. F. Chibotaru and V. H. Dao, Phys. Rev. B 81, 020502 (2010); P. J. Pereira, L. F. Chibotaru, and V. V. Moshchalkov, ibid. 84, 144504 (2011).

${ }^{17}$ E. Babaev, Phys. Rev. Lett. 89, 067001 (2002); 94, 137001 (2005); Nucl. Phys. B 686, 397 (2004); Y. Tanaka, Phys. Rev. Lett. 88, 017002 (2001); J. Phys. Soc. Jpn. 70, 2844 (2001); E. Babaev, J. Jäykkä, and M. Speight, Phys. Rev. Lett. 103, 237002 (2009).

${ }^{18}$ M. E. Zhitomirsky and V.-H. Dao, Phys. Rev. B 69, 054508 (2004).

${ }^{19}$ V. G. Kogan and J. Schmalian, Phys. Rev. B 83, 054515 (2011).

${ }^{20}$ C. Bersier, A. Floris, A. Sanna, G. Profeta, A. Continenza, E. K. U. Gross, and S. Massidda, Phys. Rev. B 79, 104503 (2009).

${ }^{21}$ A. Gurevich, Physica C 456, 160 (2007).

${ }^{22}$ A. Floris, A. Sanna, M. Luders, G. Profeta, N. N. Lathiotakis, M. A. L. Marques, C. Franchini, E. K. U. Gross, A. Continenza, and S. Massidda, Physica C 456, 45 (2007).
${ }^{23}$ A. A. Golubov, J. Kortus, O. V. Dolgov, O. Jepsen, Y. Kong, O. K. Andersen, B. J. Gibson, K. Ahn, and R. K. Kremer, J. Phys.: Condens. Matter 14, 1353 (2002).

${ }^{24}$ A. A. Shanenko, M. V. Milošević, F. M. Peeters, and A. V. Vagov, Phys. Rev. Lett. 106, 047005 (2011); L. Komendová, M. V. Milošević, A. A. Shanenko, and F. M. Peeters, Phys. Rev. B 84, 064522 (2011)

${ }^{25}$ E. Babaev and M. Speight, Phys. Rev. B 72, 180502(R) (2005); V. Moshchalkov, M. Menghini, T. Nishio, Q. H. Chen, A. V. Silhanek, V. H. Dao, L. F. Chibotaru, N. D. Zhigadlo, and J. Karpinski, Phys. Rev. Lett. 102, 117001 (2009); A. Chaves, L. Komendová, M. V. Milošević, J. S. Andrade, Jr., G. A. Farias, and F. M. Peeters, Phys. Rev. B 83, 214523 (2011).

${ }^{26}$ L. Komendová, Y. Chen, A. A. Shanenko, M. V. Milošević, and F. M. Peeters, Phys. Rev. Lett. 108, 207002 (2012).

${ }^{27}$ A. Oral, S. J. Bending, and M. Henini, Appl. Phys. Lett. 69, 1324 (1996).

${ }^{28}$ M. R. Eskildsen, M. Kugler, S. Tanaka, J. Jun, S. M. Kazakov, J. Karpinski, and Ø. Fischer, Phys. Rev. Lett. 89, 187003 (2002).

${ }^{29}$ Y. Koval, X. Y. Jin, C. Bergmann, Y. Simsek, L. Özyüzer, P. Müller, H. B. Wang, G. Behr, and B. Büchner, Appl. Phys. Lett. 96, 082507 (2010). 